\title{
QED on a momentum lattice *
}

\author{
J.B. Kogut and J.-F. Lagaë ${ }^{\dagger}$ \\ Department of Physics, University of Illinois, 1110 West Green Street, Urbana IL 61801, USA
}

We investigate the possibility of doing momentum space lattice simulations as an alternative to the conventional method. The procedure is introduced and tested for quenched QED2 and quenched QED3. Interesting physical applications to unquenched QED3 and quenched QED4 are also briefly discussed.

\section{Introduction}

The basic idea of the momentum space lattice method is very straigthforward: one just studies continuum QED on the torus (or more generally on any compact manifold). Fourier expansion then leads to a discretized problem and the number of degrees of freedom can be made finite by imposing an ultraviolet cut-off. For simplicity, we will consider here that the length $L$ of the torus (size of the box) is the same in each direction. However, finite temperature type situations can also easily be handled.

After gauge fixing (to the Feynman gauge) and Fourier expansion, the Euclidian action of QED in $\mathrm{d}$ dimensions can be recast in the form:

$$
\begin{aligned}
S & =-\frac{1}{2} \beta \sum_{q} \theta_{\mu}^{*}(q)(2 \pi q)^{2} \theta_{\mu}(q) \\
& +\sum_{k} \bar{\chi}(k)\left[\gamma_{\mu}\left(2 \pi k_{\mu}\right)+\rho\right] \chi(k) \\
& -\sum_{k, k^{\prime}} \bar{\chi}(k) \gamma_{\mu} \theta_{\mu}\left(k-k^{\prime}\right) \chi\left(k^{\prime}\right)
\end{aligned}
$$

where, instead of the continuum fields $\psi$ and $A_{\mu}$, we have used the following dimensionless variables:

$$
\chi(k)=L^{\frac{(d+1)}{2}} \psi(k) \quad \theta_{\mu}(q)=e L^{(d-1)} A_{\mu}(q)
$$

and $\beta=L^{(d-4)} / e^{2} \quad \rho=m L$. In the above formula, the $q_{\mu}{ }^{\prime} s$ take integer values and the $k_{\mu}{ }^{\prime} s$ are integers or half-integers depending on whether

\footnotetext{
*Work supported in part by the National Science Fundation under grant NSF-PHY92-00148

$\dagger$ Present address: Dept. of Physics and Astronomy, University of Kentucky, Lexington, KY 40506
}

the boundary conditions for the fermions are periodic or antiperiodic. On an $N^{d}$ lattice (三 definition) the sums over $k$ and $k^{\prime}$ are truncated in such a way that $\mathrm{N}$ positive frequency modes are included in each direction. $q$ then takes all the possible values of the momentum transfer between $k$ and $k^{\prime}$.

The method has two main advantages: 1) the coupling of the fermions to the gauge fields is noncompact and 2) there is no fermion doubling. The first is crucial for physical applications to QED3 and QED4 as will be discussed below. The second is simply a consequence of the fact that what we consider is nothing more than a particular formulation of continuum QED. The method also has two potential problems, which we should discuss here. They are: 1) The fact that the fermionic matrix is no longer sparse in momentun space (due to the convolution with the gauge field) and 2) The breaking of gauge invariance induced by imposing an ultraviolet cut-off. The first is easily dealt with by using repeated Fast Fourier Transform in matrix multiplications. This brings the computer time requirements from $O\left(N^{2}\right)$ to $O(N \ln N)$, which is still $\sim \ln N$ slower than the position space method; however, because of the non-compact character of the coupling mentioned above, smaller lattices are generally sufficient in momentum space, so that on the overall, we can still gain. The problem of gauge invariance is well known from conventional perturbation theory in quantum electrodynamics: $\Pi_{\mu \nu}$ is not transverse when computed with an ultraviolet cut-off. The cure to this problem is to use a gauge invariant regularization: for example, Pauli-Villars. In the case of QED2 and QED3, the procedure simply 
consists in dividing the usual fermionic determinant by the determinant of a heavy fermion of mass $M$ and taking the limit $\Lambda \rightarrow \infty$ (number of modes going to infinity) before letting $M \rightarrow \infty$. In 4 dimensions, the procedure is slightly more involved and would include two regularizing determinants 11. Coming back to the previous case, the implementation of the algorithm is straightforward: in the Hybrid Monte Carlo algorithm, one replaces the usual pseudo-fermion potential by $\phi^{*} A_{M}^{\dagger}\left(A_{m} A_{m}^{\dagger}\right)^{-1} A_{M} \phi$. As far as computer time is concerned, the additional multiplication by $A_{M}$ doesn't make much difference.

\section{QED2}

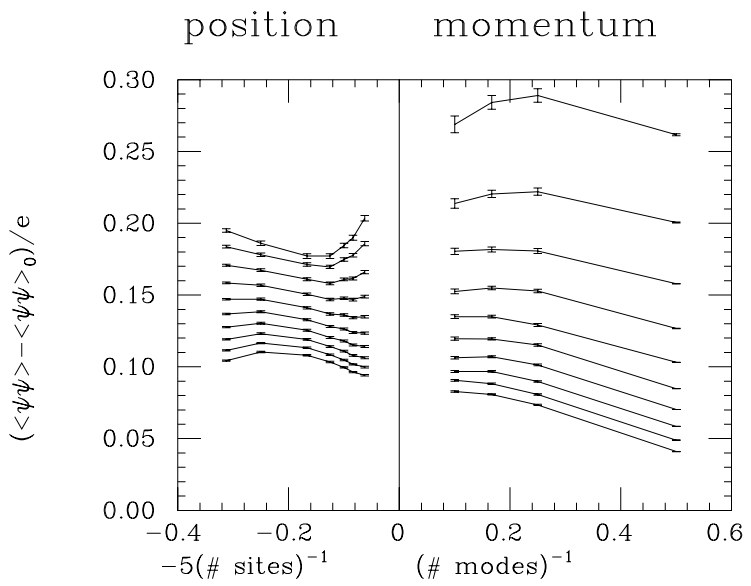

Figure 1. A comparison between position and momentum space measurements of the chiral condensate. $e L=20$ and $m / e$ ranges from 0.02 to 0.2 (from top to bottom)

Because it is computationally cheap, QED2 has been considered as a test case. We restricted our attention to the zero topological sector of the theory, i.e. configurations for which the flux of the electric field through the torus is zero (whereas in general $\phi=2 \pi n$ ). This restriction would be a drastic step in the full Schwinger model (where the chiral condensate comes from nontrivial topology [2]). However, in the quenched case to be discussed here, the theory resulting from this approximation remains interesting. We measured both $\langle\bar{\psi} \psi\rangle$ and the mass gap on lattices of various "physical size" $(\mathrm{eL}=20,30,40)$ for various number of modes $(2 \times 2,4 \times 4,6 \times 6,10 \times 10)$ and for various values of the bare fermion mass (ranging from $m / e=0.02$ to $m / e=0.2$ ). Some of our results for $\langle\bar{\psi} \psi\rangle$ are compared on fig. 1 with those obtained from the position space non-compact formulation of QED2. Although in both cases the extrapolation to an infinite ultraviolet cut-off appears rather difficult, an agreement between the two methods seems possible. It would certainly be interesting to investigate this in greater detail particularly in relation with the claim [3] that the chiral condensate should diverge in the quenched Schwinger model (remember that we have only the zero topological sector here). We have also measured the mass gap in this model. The results are presented in Fig. 2 in the form of $M(p) /|p|$ where $M$ is the dynamical fermion mass and $\mathrm{p}$ is the lowest momentum available on the lattice $\left(p_{0}=\pi / L, p_{1}=0\right)$. As one should expect, the results converge faster with increasing number of modes on the smaller lattices. However, the signal for chiral symmetry breaking becomes better and better as the physical size of the lattice is increased. Extremely good signals for chiral symmetry breaking, are in fact a general feature of the momentum space lattice method, as will be verified below.

\section{QED3}

As is well known, the breaking of chiral symmetry in unquenched or finite temperature QED3 relies entirely on weak long range interactions. As a consequence, conventional lattice studies of this problem have been plagued by strong finite size effects [4] and quantitative measurements of the critical temperature or a determination of the phase diagram as a function of the number of flavors have remained beyond reach. The difficulty essentially comes from the fact that since large lattices are needed and the number of sites is limited by computer requirements, the lattice spacing $a$ is necessarily large and one falls inevitably into the strong coupling regime (remember that $\beta^{-1}=e^{2} a$ in $(2+1)$ dimensions). The interest of 


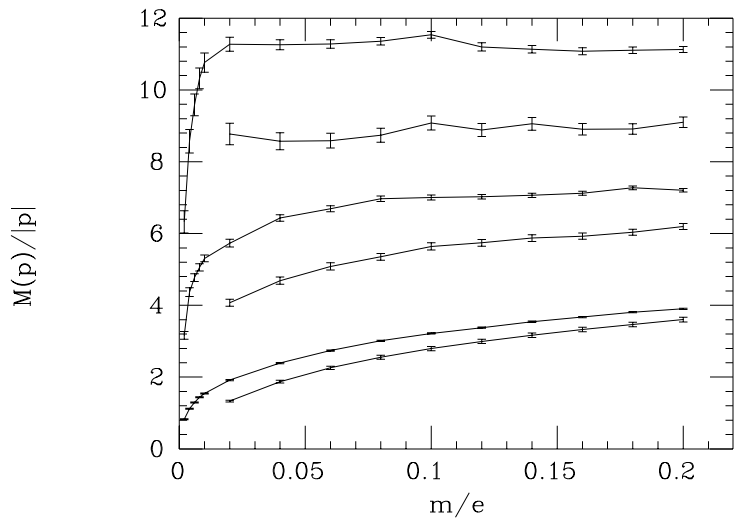

Figure 2. Dynamical versus bare fermion mass on $2 \times 2$ and $6 \times 6$ lattices of size $\mathrm{eL}=40$ (top), 30 (middle) and 20 (bottom)

applying the momentum space lattice method to this problem, comes from the fact that, in this formulation, the coupling of the fermions to the gauge field is non-compact. Therefore, one can increase the "physical size" of the lattice while remaining relatively close to the continuum limit. Our preliminary results so far mainly concern the quenched case where we have checked that the method gives a very clear signal for chiral symmetry breaking : In the relevant range, the dynamical mass at zero momentum is always much larger than the bare fermion mass.

\section{QED4}

One of the most striking features of the numerical simulations of quenched non-compact QED4 is that the results do not agree with the analytical prediction of Miransky. It has been argued that this is a consequence of induced 4 -fermi interactions on the lattice and shown that the critical exponent are expected to vary continously along the critical line in the $(\alpha, G)$ plane [5]. However, until now, no procedure has been found for modifying the lattice action in such a way that the results of the simulation would move (along the critical line) in the direction of the Miransky-BardeenLeung-Love point. We would like to argue here that the momentum space lattice method is a good candidate for performing this task. The first step consists in identifying the origin of the 4 -fermi interactions on conventional lattices. An obvious candidate is revealed by performing exactly the integration over the gauge field in the path integral (which is straightforward for the non-compact action after gauge fixing). The procedure gives rise to an effective action which contains a 4-fermi interaction, a "tadpole improved" kinetic energy term, the usual current-current interaction and higher order interactions. The derivation shows clearly that the 4 -fermi term is a direct consequence of the compact character of the coupling between fermions and gauge fields and would therefore not be there on a momentum lattice. As a consequence, we expect that the momentum space method will give results which are much closer to those of Miransky or at least will allow us to check whether these results survive the introduction of vertex corrections.

\section{Conclusion}

We have seen that the procedure of momentum space lattice simulation is practical and gives a very clear signal for chiral symmetry breaking for quenched QED both in 2 and 3 dimensions. We hope to be able to report soon on physical applications to quenched QED4. The unquenched theory in lower dimensions is also currently being tested.

\section{REFERENCES}

1. C.Itzykson and J.B.Zuber, Quantum Field Theory ( McGraw Hill, New York, 1985 )

2. I.Sachs and A.Wipf, Helv. Phys. Acta 65 , $652(1992)$

3. C.P.Van Den Doel, Nucl. Phys. B[FS10] 230 (1984) 250; S.R.Carson and R.D.Kenway, Annals of Physics $\mathbf{1 6 6}, 364$ (1986)

4. J.B.Kogut and J.-F. Lagaë, in LATTICE'92, Nucl. Phys. B ( Proc. Suppl. ) 30 (1993) 737

5. A.Kocic, S.Hands, J.B.Kogut and E.Dagotto, Nucl. Phys. B347 (1990) 217 